\title{
Circadian rhythms of body temperature in shift-workers at a coalface
}

\author{
W. P. COLQUHOUN and R. S. EDWARDS \\ Medical Research Council, Applied Psychology Unit, Cambridge
}

\begin{abstract}
Colquhoun, W. P., and Edwards, R. S. (1970). Brit. J. industr. Med., 27, 266-272. Circadian rhythms of body temperature in shift-workers at a coalface. The oral temperatures of a group of coalface workers were recorded daily at intervals during their period of work underground, over a three-week cycle of day, afternoon and night shifts. The basic mean circadian rhythm of temperature observed in the group as a whole was similar in phase and in amplitude to that of a control group of young men unused to shift work. However, it appeared that the rhythm of older coalface workers differed to some extent from that of their younger colleagues, since the mean temperature in the second half of the night was significantly higher in the older men. Whether this difference is permanent, and whether it is due to age alone rather than to long experience of night-shift working, has yet to be determined.

There were indications that the changes in the mean on-shift trend in temperature during the week in response to successive days of an altered sleep-waking routine were more pronounced, in the group as a whole, than the changes observed in a control group of young men when the latter were exposed to similar unusual routines.

It is recommended that these findings be substantiated on a considerably larger number of coalface workers before their implications for shift working are considered.
\end{abstract}

It has been known for some time that many physiological processes, including the internal temperature of the body, exhibit a circadian periodicity (Mills, 1966). A recent study at the Applied Psychology Unit (APU), Cambridge has shown that body temperature rises during the day to reach its peak value at about $8 \mathrm{pm}$, and falls during the night to its lowest value at about 4 am (Blake, 1967). It has also been demonstrated at the APU that efficiency at mental tasks which measure alertness or concentration tends to follow the temperature rhythm relatively closely, even when the latter alters as a result of working at unusual times, e.g., at night. These findings have been discussed in a number of publications (Colquhoun, Blake, and Edwards, 1968a, 1968b, 1969) and would, at first sight, appear to have obvious application to shift-work. However, in the experiments at the APU the subjects were all young men, and not used to working at unusual hours. It is possible, therefore, that the results obtained were specific to the particular population studied. Before the findings on the relationship of temperature rhythm and mental efficiency can be applied to the problem of industrial shift-system design it is necessary to establish whether the temperature cycles of people who are older and/or used to shift-work differ in any significant way from those of the sample of young men investigated in the laboratory experiments.

Coal-mining has traditionally been conducted on a shift-work basis. The mining population thus contains a large number of people who are used to working at unusual hours; many of these people are, of course, relatively old also. Miners then provide a convenient group for the purposes described above. One advantage of studying miners rather than, say, factory operatives is that the environmental conditions at many coalfaces are essentially identical whatever the time of day or night; thus factors such as changes from natural to 
artificial light, alterations in ambient temperature, etc. which might, in their capacity of Zeitgebers (Aschoff, 1960), influence the results obtained from shift-workers in a surface factory, are effectively eliminated. A second advantage lies in the fact that the type of work carried out underground in coal mines varies but little from shift to shift; this is not always the case in other industries.

The main aim of the present study was to test the feasibility of obtaining temperature readings under the somewhat difficult conditions obtaining at the coalface. If it proved possible to obtain such data, it was hoped to determine by analysis of the results whether further investigations on a larger scale would be likely to yield information which would be both scientifically valuable and of practical application in the design of optimal shift-work systems.

\section{Method}

The study was carried out over three consecutive weeks on face workers at a colliery where a three-shift system was in operation. These shifts covered the following times: 0600-1400 ('day' shift); 1400-2200 ('afternoon' shift); and 2200-0600 ('night' shift). Measurements were taken at a single coalface which was operated for two of the three shifts in any one five-day week, the other shift being 'blank'. The two shifts actually operated changed each week in a way which meant that any given man worked for one week on each of the three shifts over the three-week period. Ambient temperature at the face remained between $60^{\circ}$ and $70^{\circ} \mathrm{F}\left(15^{\circ}\right.$ and $\left.21^{\circ} \mathrm{C}\right)$ throughout the study.

Temperature measurements were taken orally at predetermined intervals during the shift by means of Dependatherm electronic thermometers. One of these instruments was sited at each end of the coalface, to obviate the necessity of transporting a single instrument through the face to record the temperatures of men who remained permanently at one or other end. Two observers were thus required for each shift: these observers were senior operatives from the shift crews who volunteered to take the temperatures, and to record them, together with the hours of sleep taken in the previous 24 hours, on special forms provided.

\section{Treatment of data}

Perhaps not unexpectedly, in view of the nature of the work performed by the subjects and the conditions under which the measurements were taken, the record sheets were not as complete as they would have been in a controlled laboratory experiment. Nevertheless sufficient information was obtained to warrant at least a simple analysis of mean trends. However, there were three main difficulties in assessing these trends: (1) due to manning problems, some men had to be transferred to coalfaces other than the selected one during one or two weeks of the study; thus the samples for 'day', 'afternoon' and 'night' shifts were not composed entirely of the same individuals; (2) not all the men on a given shift at the selected coalface worked all the five shifts for that week; thus the samples for different days of a given week were also not composed entirely of the same individuals; (3) because of a certain staggering of times of arrival and departure from the coalface, and also the exigencies of the work itself, the actual times at which the temperature readings were taken varied somewhat, even among a given group of subjects on the same shift.

It was decided, for the purposes of the simple analysis which was performed, to ignore the sampling errors inherent in (1) and (2) above, and to assume that in all cases a representative set of readings was obtained. As a partial solution to the problem posed by (3) above, the data were examined to determine, for each shift, those times around which the majority of readings tended to cluster, and to collate all readings taken within 30 minutes of these times. This procedure gave the following sets of times:

'Day' shift :

'Afternoon' shift: $0700,0900,1030,1300$

'Night' shift: $1500,1630,1730,1830,2000$ $2400,0130,0230,0330,0500$

Not all the subjects who were present on any particular shift were represented at all the times in that shift, but, again, it was assumed for present purposes that at each time a representative sample was obtained.

The approximations and assumptions detailed above should be borne in mind in considering the results of the analysis which follows.

\section{The 'normal' rhythm of the group}

The first point to be examined was whether the 'normal' 24-hour temperature rhythm of the coalface workers differed in any obvious way from that of the young men studied at APU. By 'normal' here is meant the rhythm which is observed when, during the days immediately before the readings are taken, the subjects have followed a 'natural' sleep-waking routine (i.e., sleeping from (say) $11.30 \mathrm{pm}$ to 7.30 am). By definition shift workers never in fact follow such a routine except, presumably, when on holiday. Therefore, the only possible occasions on which a 'normal' rhythm could have been obtained from the present subjects during the observation period were the three Mondays, these being days which were preceded by two days of 'natural' routine during the weekends. However, since readings were available only for that section of the day during which the subjects were at the coal face, these 'normal' Monday rhythms could not be determined directly. Instead, a single composite 24-hour curve was derived by plotting the mean on-shift trends obtained from day, afternoon and night shifts on the three successive Mondays. The numbers of individuals on which these mean trends were based were: 12 (day shift); 11 (afternoon shift); and 17 (night shift). This composite curve is shown in Fig. 1, together with a curve obtained from 22 young men at the APU over the first 24 hours of a 12-day night-shift experiment.

The curves in Fig. 1 indicate that the average rhythm of the present subjects was very similar to that of the young men. Peak and trough readings occurred at similar times, and the differences 


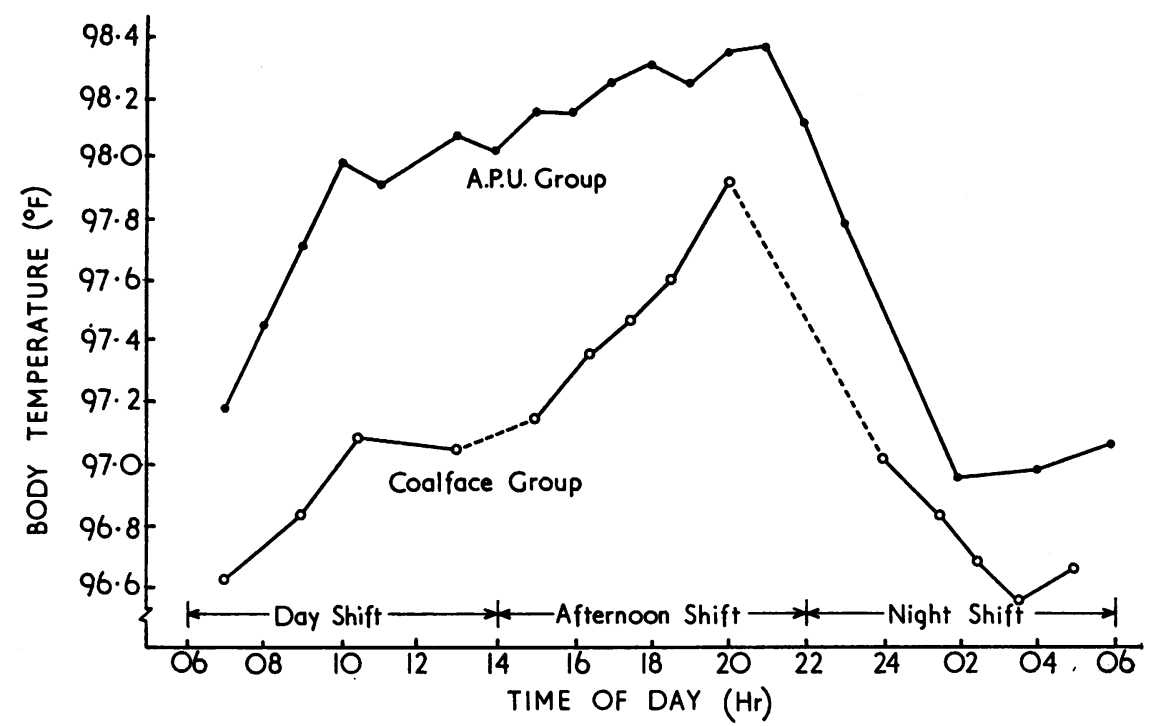

FIG. 1. Mean 'normal' 24-hour temperature curves for coalface workers and for APU subjects.

between them (i.e., the ranges) were also comparable. There was a certain difference in overall temperature level, but this was most probably due to the use of Dependatherm instruments in the present case (these thermometers are usually found to give readings which are lower than those obtained from the standard clinical thermometers which were used with the APU subjects). Thus it would appear that there is no obvious difference in the 'normal' rhythms of coalface shiftworkers and young men who are not used to working shifts.

Changes in on-shift temperatures during the week When subjects in the APU studies were made to work at unusual hours for a period of 12 consecutive days, it was found that the temperature rhythm changed in response to whatever new sleep-waking cycle was imposed. However, relatively little change was evident in the first five days; thus it is of considerable interest to determine whether there was any evidence of more rapid change in the present group of men who were used to such unusual routines. That temperature rhythms do in fact adapt more rapidly to night work in shift-workers is suggested by the work of van Loon (1963).

Day shift In one experiment at APU the subjects were required to work an 'early' shift from 0400 to 1200 every day; they thus had to get up well before the time that was usual for them. It was found that the temperature rhythm altered in response to this different routine in such a way that the rise in temperature to be expected over the hours during which this shift was worked increased in magnitude as the trial progressed, but only very slowly (Colquhoun et al., 1968b). Although the day shift began two hours later than the APU 'early' shift, it was nevertheless also necessary for the present subjects, during their week on 'days', to get up at an earlier time than they would probably otherwise have done. The daily records were therefore examined to determine whether the on-shift temperature trend altered during the week on day shift in a way comparable to that of the APU subjects, and, if so, whether the rate of change was more rapid. The answer appeared to be affirmative on both counts; comparison of the trends for Monday and Friday (see Fig. 2) indicates a considerable increase in the on-shift rise in temperature, of an extent observed in the APU subjects only when the latter had been working their 'early' shift for 12 consecutive days. The difference in mean temperature on Monday and Friday at the 1300 reading time was found to be highly significant statistically $(\mathrm{P}<0.005)$.

Thus it would appear that the average temperature rhythm of the coalface workers did alter in response to the slightly abnormal sleep-waking routine demanded by work on the day shift, and that the rate of change was probably faster than would be expected from non-shift-workers exposed to the same routine.

Afternoon shift Since this shift did not entail any alteration in 'normal' sleeping hours, it was not expected that any alteration in temperature rhythm would occur during the week. Examination of the daily on-shift trends suggested that this was in fact the case; the trends for Monday and Friday are plotted in Figure 3.

Despite somewhat more irregularity in the Friday 


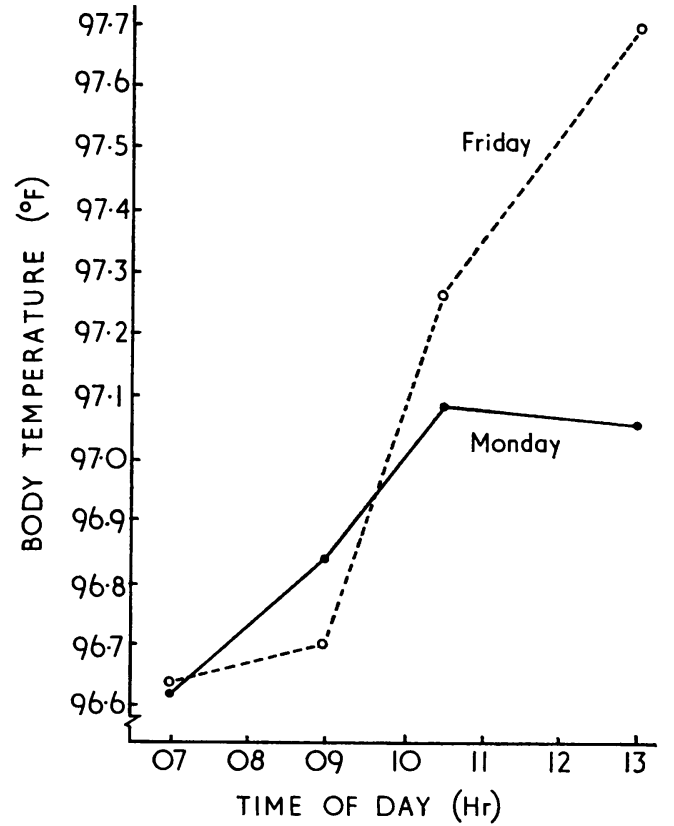

FIG. 2. Mean on-shift trends in the temperature of coalface workers on Monday and Friday of the day shift week.

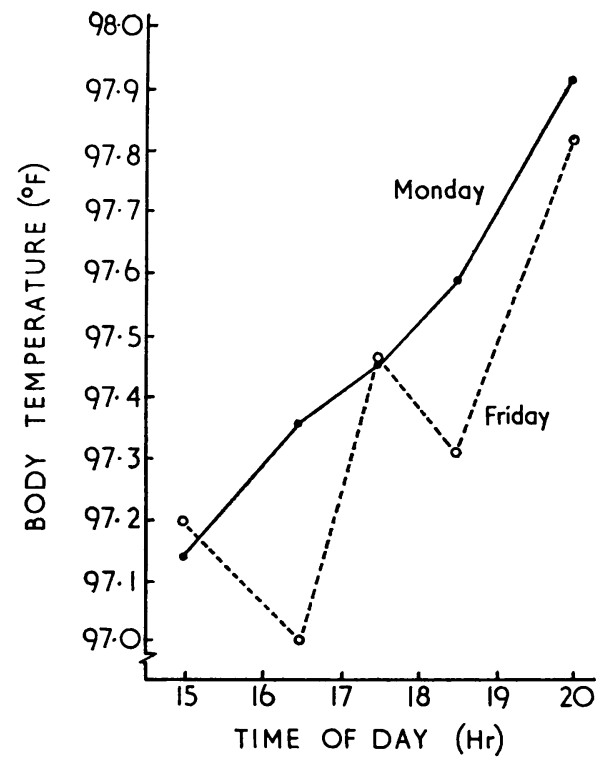

FIG. 3. Mean on-shift trends in the temperature of coalface workers on Monday and Friday of the afternoon shift week. trend, at no recording time did the mean temperature on the two days differ significantly.

Night shift In many ways this shift is the most critical of the three, since it demands a radical alteration in sleeping hours. When APU subjects were put on to a shift identical with one worked by the present group, it was found that the overall mean temperature recorded during the shift rose steadily from night to night over the first five nights. However, there was little noticeable change in the on-shift trend in temperature until about the sixth night. From this point on this trend became progressively flatter, and it was this flattening that was reflected in measures of mental efficiency (Colquhoun et al., 1968b).

If the temperature rhythm does indeed adapt more rapidly to unusual routines in shift-workers than in non-shift-workers (as the results for the day shift would seem to confirm), then it might be expected that the on-shift flattening of the temperature trend in the present group would appear at an earlier stage, and would therefore be clearly noticeable by the Friday of the night shift week.

Unfortunately, readings for only seven subjects (mostly young) were available for the Friday of the night shift week. It was felt that, because of the possibility that this group was unrepresentative of the men as a whole, the results for Friday could not legitimately be used to test this supposition. Therefore the extent of within-week change was examined by a comparison of the trends on Monday and Thursday. These trends are shown in Fig. 4, together with the mean trends of the 10 APU subjects who worked the same shift, on the corresponding days of their own trial period.

It will be seen that although the overall temperature value, as in the case of the APU subjects, rose between Monday and Thursday, the extent of the increase was much smaller and was not statistically significant. Although there was no clear indication of flattening in the overall on-shift trend, the fall in temperature during the first half of the shift which was evident on Monday had disappeared by Thursday. The change in temperature between 2400 and 0230 was in fact significantly different on the two days $(\mathrm{P}<0.02)$. Thus at least a partial flattening of the curve had occurred, and to this extent the prediction that adaptation would be more rapid in the present group of subjects was confirmed.

\section{Analysis by age groups}

The age of the subjects ranged from 26 to 62 , with a mean of 45.7 and a median of 47.5 years. In order to discover whether there was any difference in the temperature rhythms of younger and older men, mean trends were assessed separately for those of 


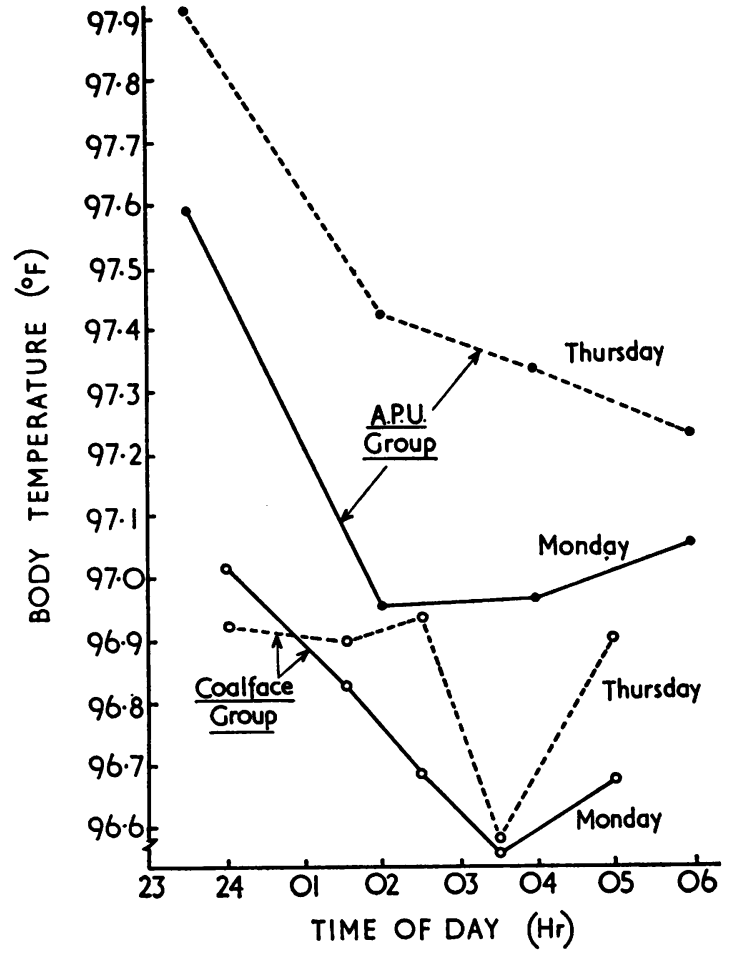

FIG. 4. Mean on-shift trends in the temperature of coalface workers and APU subjects on Monday and Thursday of the night shift week.
46 years and below, and those of 47 and above.

The composite 'Monday' rhythms of the younger and older groups are shown in Figure 5. The curves suggest that the older group had a lower temperature at the start of the day shift, a higher temperature at the end of the afternoon shift and a higher temperature throughout the second half of the night shift. However, only the difference in the night shift was statistically significant $(P<0.05)$ when the readings for 0230,0330 and 0500 were combined. A striking aspect of the night shift results was that not only the overall level, but also the on-shift trend, appeared to differ in the two groups, the curves for the second half of the shift being almost mirror images of each other. These differences remained constant on each of the four nights of the week on which a comparison could be made (see Fig. 6, in which the readings for Monday and Thursday are shown for illustration).

The difference between night-shift temperatures in younger and older workers is of considerable interest, since it implies that the basic temperature rhythm may be partially altered by extensive exposure to night-shift working. However, further trials would be necessary to substantiate this conclusion, since the present samples were inevitably rather small. These trials would, of course, have to include measurement of the rhythms of older nonshift-workers also, in order to check that the alteration was not due to age alone; they should also include measurements of the rhythms of coalface workers who had not worked shifts in the recent

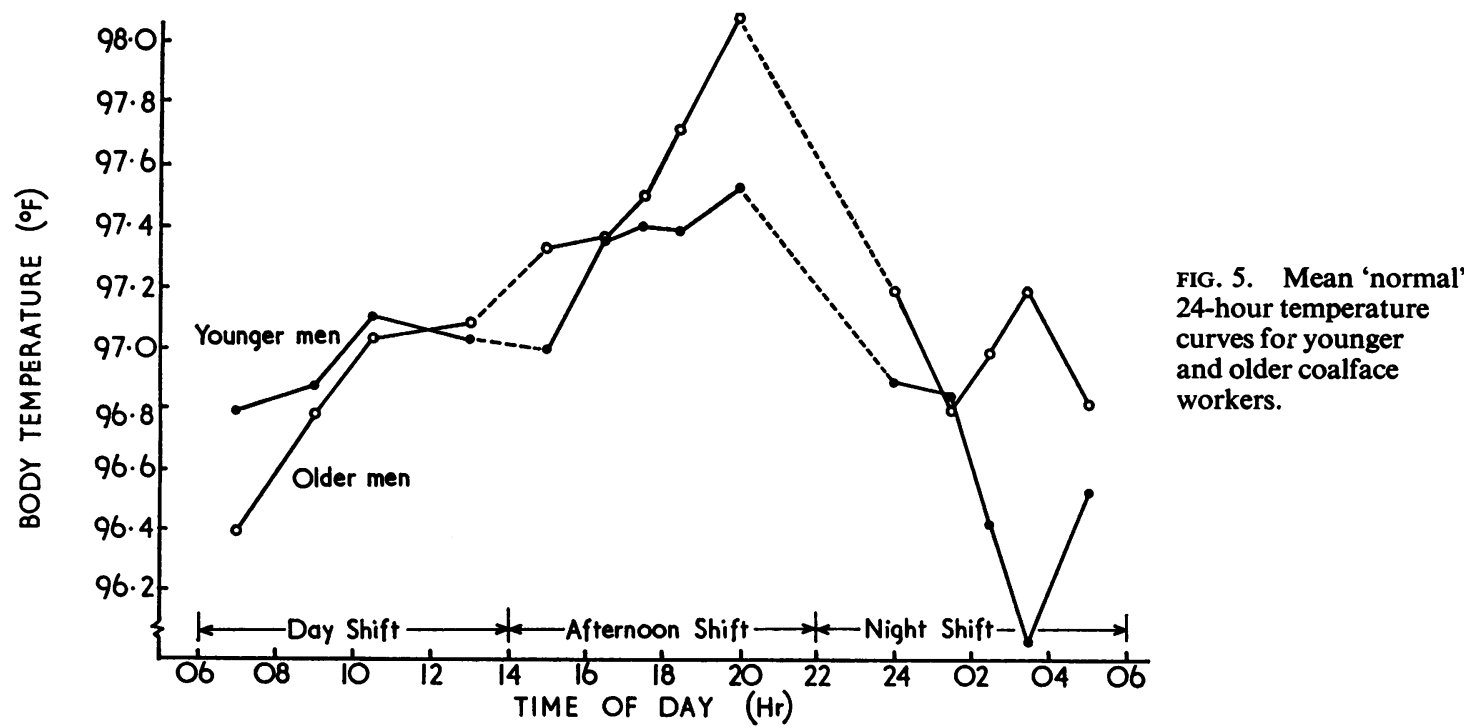




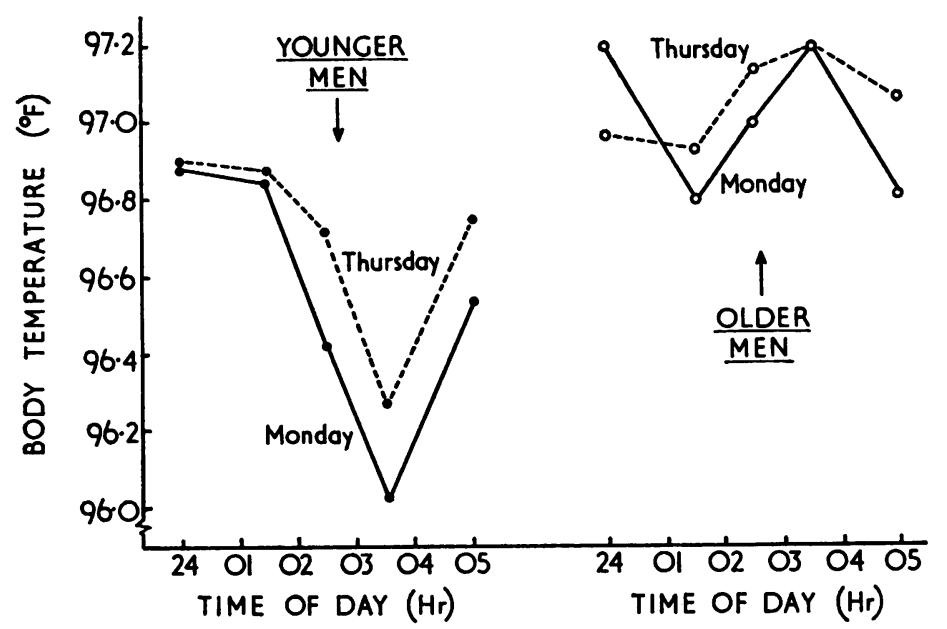

FIG. 6. Mean on-shift trends in the temperature of younger and older coalface workers on Monday and Thursday of the night shift week. past, to determine whether the alteration was reversible.

\section{Individual differences}

As expected, there were considerable individual differences in the extent of the on-shift temperature changes observed. As an illustration of the magnitude of these differences the range of the values recorded on each of the three shifts is given in the Table for the first and last shift days of the week.

When allowance is made for the effects of age already discussed, the range of variation evident in the Table is comparable with that found in the APU studies of young men. It will be noted that in all cases bar one the range includes values which are actually opposite in sign to the mean. In the night

\section{TABLE}

Means and Ranges of Observed Temperature Changes $\left({ }^{\circ} \mathrm{F}\right)$ DURING The Day, Afternoon and Night SHIFTs

(Values shown are the differences between the initial and final readings on the first and last day of the shift week.)

\begin{tabular}{|c|c|c|c|c|c|c|}
\hline & \multicolumn{2}{|c|}{ Day shift } & \multicolumn{2}{|c|}{ Afternoon shift } & \multicolumn{2}{|c|}{ Night shift } \\
\hline & Mean & Range & Mean & Range & Mean & Range \\
\hline $\begin{array}{l}\text { First } \\
\text { shift day1 }\end{array}$ & +0.43 & $\begin{array}{l}-0.6 \\
\text { to } \\
+1.0\end{array}$ & +0.78 & $\begin{array}{l}-0.2 \\
\text { to } \\
+1.4\end{array}$ & -0.55 & $\begin{array}{l}-2.0 \\
\text { to } \\
+1.0\end{array}$ \\
\hline $\begin{array}{l}\text { Last } \\
\text { shift day }{ }^{2}\end{array}$ & $+1 \cdot 03$ & $\begin{array}{l}+0.2 \\
\text { to } \\
+1.8\end{array}$ & +0.62 & $\begin{array}{l}-0.6 \\
\text { to } \\
+1.6\end{array}$ & -0.02 & $\begin{array}{l}-0.7 \\
\text { to } \\
+1.0\end{array}$ \\
\hline
\end{tabular}

${ }^{1}$ Monday, for all shifts.

${ }^{2}$ Friday for day and afternoon shifts, Thursday for night shift. shift this would be expected, since on the Thursday partial adaptation had occurred, and on both days the older subjects were exhibiting a trend which was characteristically different from the 'norm' anyway. In the remaining cases, the actual number of subjects showing an 'incorrect' trend was very small (one only on the first day of the day and afternoon shifts, and two on the last day of the afternoon shift). Nevertheless the existence of these 'aberrant' individuals is of considerable interest.

\section{Sleep hours}

The mean number of hours of sleep per day reported was 5.6 (day shift), 8.9 (afternoon shift) and 7.8 (night shift). The relatively short sleep period when on day shift reflected the fact that the universal pattern was to go to sleep at a 'normal' hour (about $11.30 \mathrm{pm})$ and get up early. The relatively long period when on afternoon shift reflected a tendency to 'lie in' in the mornings. Although the actual number of hours spent sleeping when on night shift was comparable with the number of hours of sleep taken by the adult population as a whole in their normal lives, it was clear from examination of the records (1) that the sleep was frequently broken, i.e., it was composed of two or more separate periods; (2) that the variability in number of hours slept on successive days was considerable; and (3) that the particular times of day during which the sleep was taken differed widely in individual members of the group. These findings are typical of those reported in other studies of the sleep of night shiftworkers (Tune, 1968). There was no noticeable difference between younger and older subjects in the average number of hours slept when on a particular shift, nor in the extent to which the sleep period was broken or in the variability of the sleep pattern itself when on night shift. 


\section{Comments}

Before the scientific import of these findings is considered, and any application to the design of optimal shift-work systems for coalface workers is contemplated, they should be substantiated on a considerably larger number of men, of all age groups, and including, if possible, some people who are assigned to 'permanent' shifts as well as others who follow a 'rotating' system. Particular attention should be paid to the nature and extent of individual differences both in the 'basic' temperature rhythm and in the degree to which this is changed by working at unusual hours.

Despite the difficulties involved, it is important that in any future study of temperature rhythms in coalface workers an attempt should be made (1) to obtain readings off-shift as well as when actually working; (2) to standardize the intervals between successive readings; and (3) to ensure, as far as possible, that the subjects are present on all occasions on which readings are due to be taken.

Acknowledgments are due to the National Coal Board, the National Union of Mineworkers and the Ministry of Technology for facilitating this study. We are particularly indebted to the staff of the Medical Department, N.C.B. (South Nottinghamshire Area), the Manager and staff of Linby Colliery, the officers of the N.U.M. (Linby Branch) and Mr. J. K. L. Thompson, Senior Regional Officer, Mintech. (East Midlands). Our special thanks go to the individual workers at Linby Colliery from whom the temperature measurements were obtained; without their assistance this study would not have been possible.

\section{References}

Aschoff, J. (1960). Exogenous and endogenous components in circadian rhythms. Cold Spr. Harb. Symp. quant. Biol., 25, 11-28.

Blake, M. J. F. (1967). Relationship between circadian rhythm of body temperature and introversion-extraversion. Nature (Lond.), 215, 896-897.

Colquhoun, W. P., Blake, M. J. F., and Edwards, R. S. (1968a). Experimental studies of shift-work I: A comparison of 'rotating' and 'stabilized' 4-hour shift systems. Ergonomics, 11, 437-453. -1 (1968b). Experimental studies of shift-work II Stabilized 8-hour shift systems. Ergonomics, 11, 527-546. , - - (1969). Experimental studies of shift-work III Stabilized 12-hour shift systems. Ergonomics, 12, 865-882.

van Loon, J. H. (1963). Diurnal body temperature curves in shift workers. Ergonomics, 6, 267-273.

Mills, J. N. (1966). Human circadian rhythms. Physiol. Rev., 46, $128-171$.

Tune, G. S. (1968). The human sleep debt. Sci. J., 4, pp.67-71.

Received for publication November 28, 1969. 